\title{
REMARK ON LOOP SPACES
}

\section{P. J. HILTON ${ }^{1}$}

In [1] the authors prove two theorems on polyhedra with homology of finite type.

THEOREM A. If $(X, \mu)$ is a space with comultiplication, if $X$ is $(q-1)$-connected and $\operatorname{dim} X \leqq 3 q-3$, then $(X, \mu)$ is equivalent to a suspension structure.

Theorem B. If $\operatorname{dim} A \leqq 3 q-2$ and $B$ is $(q-1)$-connected, then every homomorphism $\Sigma A \rightarrow \Sigma B$ is in a suspension class.

In this note we make some remarks on the duals of these theorems. The dual of Theorem A reads as follows (we need here no assumption on the homology of $X$ ).

THEOREM C. If $(X, \mu)$ is a space with multiplication, if $X$ is $(q-1)$ connected, $q>1$, and $\pi_{n}(X)=0$ for $n \geqq 3 q$, then $(X, \mu)$ is equivalent to a loop-space structure.

This theorem may be proved as an application of Stasheff's theory of $A_{n}$-spaces, and has certainly been noted by Stasheff. A method of proof was outlined in [3] in order to prove Corollary 3.12 of that paper, but the proof was defective. ${ }^{2}$ We give here a proof whose structure is essentially dual to that of the structure of the proof of Theorem A in [1], but which is much simpler in detail. Just as in Theorem A we presented $X$ as the suspension of a (generalized) homology section of its coprojective plane, so here we present it as the loop space of a homotopy section of its projective plane.

The dual of Theorem B is Sugawara's Theorem (7.4 of [8]).

Theorem D. If $\pi_{n}(A)=0, n \geqq 3 q-1$, and $B$ is $(q-1)$-connected, $q>1$, then every homomorphism $\Omega B \rightarrow \Omega A$ is homotopic to a loop map.

It turns out, similarly, that the dual version of the proof of Theorem B is much simpler than the proof of Theorem B itself, owing to a fortuitous circumstance which we discuss at the end of this note.

Received by the editors April 12, 1963.

1 The author was supported by NSF grant G 15984 during the preparation of this note.

2 This came to light when I. Berstein noticed the corresponding defect in an early version of the proof of Theorem $A$. 
In the proofs of Theorems $\mathrm{C}$ and $\mathrm{D}$ we use the Blakers-Massey Theorem stated in terms of the fibre and cofibre of an arbitrary map. It seems to us that this formulation might have some independent interest. It has long been recognized (see, e.g., [5]) that the Serre Theorem (Corollary 1, p. 469 of [6]) is dual to the Blakers-Massey Theorem, so we set it down here alongside the Blakers-Massey Theorem, using the same formulation, although we have no need of it in proving Theorems $\mathrm{C}$ and $\mathrm{D}$.

Let $f: P \rightarrow Q$ be a map with fibre $F$ and cofibre $C$. We write this as a sequence

$$
F \stackrel{i}{\rightarrow} P \stackrel{f}{\rightarrow} Q \stackrel{j}{\rightarrow} C .
$$

Associated with (1) there is a map $u: F \rightarrow \Omega C$ with adjoint $\bar{u}: \Sigma F$ $\rightarrow C ; \bar{u}$ may also be regarded as dual to $u$. To describe $u$ it is sufficient to consider the case when $f$ is a cofibration inclusion. Then $C=Q / P$ and $F=E\left(Q ; P,{ }^{*}\right)$, the space of paths on $Q$ beginning in $P$ and ending at the base point. Then the identification map $j: Q \rightarrow C$ plainly induces $u: F \rightarrow \Omega C$.

We may now state the Blakers-Massey and Serre Theorems in terms of (1) and the associated maps $u, \bar{u}$.

Proposition 2. Suppose in (1) that $f$ is $(k-1)$-connected and $P$ is $(l-1)$-connected, $k \geqq 2, l \geqq 1$. Then $u: F \rightarrow \Omega C$ is $(k+l-2)$-connected.

Proposition 3. Suppose in (1) that $f$ is $(k-1)$-connected and $Q$ is $(l-1)$-connected, $k \geqq 3, l \geqq 2$. Then ${ }^{3} \bar{u}: \Sigma F \rightarrow C$ is $(k+l-1)$-connected.

Of course, Proposition 3 differs in form very much from the original statement of Serre's Theorem (Corollary 1, p. 469 of [6]); we have included it here in this form to demonstrate its relation to Proposition 2 which closely resembles the original Blakers-Massey Theorem (Theorem II of [2]). Serre's Theorem actually relates the homology of $i$ and $Q$ in (1) and yields Proposition 3 through the transgression square; see also Proposition 3 of [4].

We now consider the Sugawara fibration [7] of an $H$-space $(X, \mu)$. This is a map $h: \Sigma(X \# X) \rightarrow \Sigma X$ with fibre $X$ and cofibre $X P(2)$, the projective plane of $X$. Thus we have the special case of (1),

$$
X \stackrel{i}{\rightarrow} \Sigma(X \# X) \stackrel{h}{\rightarrow} \Sigma X \stackrel{j}{\rightarrow} X P(2) .
$$

${ }^{3}$ We may take $k \geqq 2$ if we are content with the homology-connectedness of $u$. [Added in proof. These propositions, and other results discussed in this note, have been strengthened or generalized by Ganea; see his forthcoming paper, A generalization of the homology and homotopy suspension.] 
Then (4) has two features of great importance to us in the proof we give of Theorem $C$. First we have $i \simeq 0$. Second, the connecting map $\Omega \Sigma X \rightarrow X$ of the fibration $h$ is a left-inverse of the canonical embedding $e: X \rightarrow \Omega \Sigma X$. We deduce that if $u: X \rightarrow \Omega X P(2)$ is associated with (4), then (up to homotopy)

$$
\bar{u}=j: \Sigma X \rightarrow X P(2) .
$$

In fact, (5) is just a special case of the more general

Proposition 6. If, in (1), $i \simeq 0$ then the connecting map $\Omega Q \rightarrow F$ has a left-inverse $s: F \rightarrow \Omega Q$ and $u \simeq(\Omega j) s: F \rightarrow \Omega C$.

Proof. Consider the diagram

$$
\begin{aligned}
& \Pi_{1}(A, Q) \stackrel{J}{\rightarrow} \Pi_{1}(A, f) \stackrel{\epsilon}{\leftarrow} \Pi(A, F) \\
& \nwarrow j_{*} \quad \downarrow \eta \swarrow \nwarrow u_{*} \\
& \Pi_{1}(A, C)
\end{aligned}
$$

where $J$ belongs to the homotopy sequence of $f, \epsilon$ is the excision isomorphisn, and $\eta$ is induced by the map

$$
\begin{aligned}
& P \rightarrow^{*} \\
& \downarrow f \quad \downarrow \\
& Q \underset{j}{\rightarrow} C .
\end{aligned}
$$

Then $u$ is defined so that the right-hand triangle commutes and it is trivial that the left-hand triangle commutes. Also $\epsilon^{-1} J$ is induced by the connecting map.

Now suppose $i \simeq 0$. Then if $1 \in \Pi(F, F), \epsilon(1)=J\{s\}$, where $s: F \rightarrow \Omega Q$ is a left inverse of the connecting map and $j_{*}\{s\}=\eta \epsilon(1)=\{u\}$, proving the proposition.

We have only to recall that $j(=u): X \rightarrow \Omega X P(2)$ is a homomorphism to complete the proof of Theorem C. For if $X$ is $(q-1)$-connected then in (4) $h$ is $q$-connected and $\Sigma(X \# X)$ is $2 q$-connected. Thus $\bar{j}: X \rightarrow \Omega X P(2)$ is $3 q$-connected by Proposition 2 . Let $Z$ be the $3 q$ homotopy section of $X P(2)$; thus there is a map $p: X P(2) \rightarrow Z$ such that

$$
\begin{aligned}
p_{*}: \pi_{i}(X P(2)) & \cong \pi_{i}(Z), & & i \leq 3 q, \\
\pi_{i}(Z) & =0, & & i>3 q .
\end{aligned}
$$

Then $(\Omega p) \bar{j}: X \rightarrow \Omega Z$ is certainly a homomorphism of $H$-spaces; and if 
$\pi_{n}(X)=0, n \geqq 3 q$, then $(\Omega p) j$ induces an isomorphism of all homotopy groups and so is a homotopy equivalence. This completes the proof of Theorem C.

REMARK. We can improve Theorem $\mathrm{C}$ slightly by allowing $\pi_{3 q}(X)$ $\neq 0$, provided that it is free abelian. We do not know the best possible result here (as we do in the dual case); if we take

$$
X=K\left(Z_{3}, 3 ; Z_{3}, 11 ;(\beta u)^{3}\right)
$$

where $u$ is the fundamental class in $H^{3}\left(Z_{3}, 3 ; Z_{3}\right)$ and $\beta$ is the Bockstein operator then $X$ is not equivalent to a loop space, $X$ is 2-connected, so that $q=3$, and $\pi_{n}(X)=0$ for $^{4} n \geqq 12=3 q+3$.

We turn now to the proof of Theorem D. If we take $X=\Omega A$ then the fibration $h: \Sigma(\Omega A \# \Omega A) \rightarrow \Sigma \Omega A$ in (4) is induced by the canonical map $e: \Sigma \Omega A \rightarrow A$. Thus we have, up to homotopy, a fibration

$$
\Sigma(\Omega A \# \Omega A) \stackrel{h}{\rightarrow} \Sigma \Omega A \stackrel{e}{\rightarrow} A .
$$

Now if $\phi: X \rightarrow Y$ is a homomorphism of $H$-spaces, then the diagram

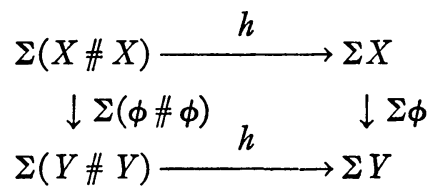

is homotopy-commutative. Thus if $\phi: \Omega B \rightarrow \Omega A$ is a homomorphism we have a diagram

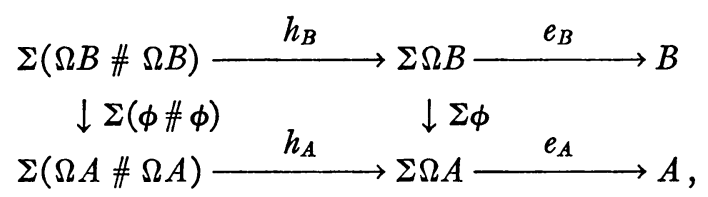

in which the rows are fibration sequences and the square homotopycommutes. It follows that the map $e_{A}(\Sigma \phi): \Sigma \Omega B \rightarrow A$ is nullhomotopic on the fibre of $e_{B}$. Now if $B$ is $(q-1)$-connected then $\Sigma(\Omega B \# \Omega B)$ is $(2 q-2)$-connected. Thus Theorem 1 of [4] enables us to infer immediately that if $\pi_{n}(A)=0, n \geqq 3 q-1$, then there exists a map $f: B \rightarrow A$ with $f\left(e_{B}\right) \simeq e_{A}(\Sigma \phi)$. That is, $\phi \simeq \Omega f$ and Theorem $\mathrm{D}$ is proved.

The simplicity of the proof of Theorem $\mathrm{D}$ rests on the fact that the fibre of $e: \Sigma \Omega A \rightarrow A$ is a functor of $\Omega A$, together with the fact that the

\footnotetext{
${ }^{4} \mathrm{~W}$. Browder has shown the author that $K\left(Z_{3}, 3 ; Z_{9}, 10 ; \lambda u(\beta u)^{2}\right)$, where $\lambda$ is induced by the coefficient homomorphism $Z_{3} \subseteq Z_{9}$ is an $H$-space but not a loop space. This narrows the gap by one dimension.
} 
homomorphic property of $\phi$ is reflected in the commutativity of the square in (8). However in the dual problem we do not seem to have so simple a situation. We constructed in [1], for any $H^{\prime}$-space $X$, a map

$$
h^{\prime}: \Omega X \rightarrow \Omega(X b X)
$$

with the property that if $\phi: X \rightarrow Y$ is a homomorphism of $H^{\prime}$-spaces then the diagram

$$
\begin{aligned}
& \Omega X \stackrel{h^{\prime}}{\longrightarrow} \Omega(X b X)
\end{aligned}
$$

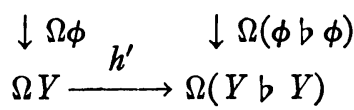

homotopy-commutes. However, if $X=\Sigma A$ then $h^{\prime}$ does not appear to be the cofibre projection associated with the canonical embedding $e: A \rightarrow \Omega \Sigma A$, and we were obliged to demonstrate in [1] that $h^{\prime}$ induces a map from the cofibre of $e^{\prime}$ to $\Omega(\Sigma A b \Sigma A)$ which is sufficiently highly connected to yield a proof of Theorem B. It appears then natural to raise the questions whether the cofibre of $e^{\prime}$ is a functor of $\Sigma A$ and how far it can differ in homotopy type from $\Omega(\Sigma A b \Sigma A) .^{5}$

\section{REFERENCES}

1. I. Bernstein and P. J. Hilton, On suspensions and comultiplications, Topology 2 (1963), 73-82.

2. A. L. Blakers and W. S. Massey, The homotopy groups of a triad. II, Ann. of Math. (2) 55 (1952), 192-201.

3. T. Ganea, P. J. Hilton, and R. P. Peterson, On the homotopy-commutativity of loop-spaces and suspensions, Topology 1 (1962), 133-141.

4. P. J. Hilton, On excision and principal fibrations, Comment. Math. Helv. 35 (1961), 77-84.

5. I. Namioka, Maps of pairs in homotopy theory, Proc. London Math. Soc. (3) 12 (1962), 725-738.

6. J. P. Serre, Homologie singulière des espaces fibrés. Applications, Ann. of Math. (2) 54 (1951), 425-505.

7. M. Sugawara, On a condition that a space is an H-space, Math. J. Okayama Univ. 6 (1957), 109-129.

8. - On the homotopy-commutativity of groups and loop spaces, Mem. Coll. Sci. Univ. Kyoto, Ser. A. Math. 33 (1960), 257-269.

Cornell University

- Added in proof. An example due to Barratt shows that the cofibre of $e^{\prime}$ is not a functor of $\Sigma A$. The second question is dealt with by Ganea (loc. cit.). 